\title{
Planung, Vorbereitung und Nachkontrolle bei Operationen von degenerativen Erkrankungen der Wirbelsäule
}

\author{
Martin Lorenz, Eckhard Rickels, Kathrin König
}

\section{Zusammenfassung}

Die Problematik der degenerativen Wirbelsäulenleiden ist in der ärztlichen Praxis ein häufiges Vorkommnis. Bei einem ansteigenden Altersdurchschnitt der Patienten und der Bereitschaft, auch in hohem Alter operativ einzugreifen, ist eine weitere Zunahme der Bedeutung abzusehen. Die moderne Radiologie ermöglicht eine genaue morphologische Darstellung der Läsionen. Diese Befunde müssen jedoch mit der Klinik korreliert werden. Bei jüngeren Patienten überwiegen monokausale Veränderungen, wie z. B. ein Bandscheibenvorfall. Bei Älteren kommt es immer mehr zu einem Mischbild von knöchernen, ligamentären und diskogenen Veränderungen mit häufig multisegmentaler Ausbreitung. Entlang der Wirbelsäule sind die Veränderungen der LWS zahlenmäßig im Vordergrund. Die häufigste Ursache, die zur Operation führt, besteht bei Kompressionssyndromen. Zur Beurteilung der OP-Indikation müssen im Vorfeld eine möglichst enge Abgrenzung von beschwerdeauslösenden Veränderungen und insbesondere auch die Komorbiditäten beachtet werden. Auch diese können das Nutzen-Risiko-Verhältnis nachhaltig beeinflussen. Gleichzeitig macht der Kostendruck im Gesundheitswesen eine möglichst ökonomische Behandlung sinnvoll mit Verlagerung von wesentlichen Anteilen der Diagnostik in den prästationären Bereich.

\section{Planning, Preparation and Follow-Up of Operations for Degenerative Diseases of the Spine}

The frequency of consultations concerning degenerative alterations of the adult spine is increasing in medical practice. The age of the patients is also increasing as well as the willingness to treat even aged persons. Radiological examinations reveal detailed information of the lesions. However, a close correlation to the clinical findings is mandatory. In younger people typically circumscribed lesions appear, such as a disc herniation, for example. Degenerative changes of the spine produce a mixture of bony, ligamentous and discogenetic disorders and coact frequently in multilevel affections with a predominance in the lumbar region. In the majority of cases the symptoms are caused by neural compression. The indication for surgery should respect the plausibility of symptoms and radiological findings as well as comorbidities, which may have an effect on the relationship between risks and benefit. The cost pressure in the public health services leads to a shifting of an essential percentage of the diagnostic procedures into the prehospital phase.

\section{Einleitung}

Die Probleme mit degenerativen Erkrankungen der Wirbelsäule gewinnen von Jahr zu Jahr an Bedeutung. Die Anzahl von Patienten mit chronischen Schmerzen in diesem Bereich hat in den letzten 20 Jahren stetig zugenommen. 2008 wurden über 512000 Patienten unter der betreffenden ICD stationär behandelt, etwa die Hälfte davon war über 65 Jahre alt [10]. Im Verlauf eines Jahres klagen 62\% der Frauen und 56\% der Männer über Rückenschmerzen, wobei allerdings in einem überwiegenden Anteil eine spontane Besserung unter konservativer Behandlung gelingt. Nur etwa 15\% aller Rückenschmerzen sind spezifische Rü-

OP-JOURNAL 2011; 27: 34-40

(C) Georg Thieme Verlag KG Stuttgart · New York DOI http://dx.doi.org/10.1055/s-0030-1271095 ckenschmerzen, deren Ursache speziell behandelt werden muss [2]. Die Ausgaben für stationäre Leistungen bei der Behandlung von Rückenerkrankungen (ICD-9 720-724) betrugen bereits 1999 2,5 Milliarden DM, insgesamt wurden die Kosten für die Behandlung, Rehabilitation und vorzeitige Berentung von Patienten mit Rückenschmerzen in diesem Zeitraum auf über 30 Milliarden DM jährlich geschätzt; die direkten und indirekten Krankheitskosten auf etwa 50 Milliarden DM [2].

Bei den operativen Behandlungen stehen die Nukleotomien im Vordergrund. 2009 wurde die assoziierte DRG 5-831 (Exzision von erkranktem Bandscheibengewebe) über 161000 -mal verschlüsselt und nimmt somit zahlenmäßig den Platz 15 bei den vollstationären Operationen [10] ein. Das Vorkommen eines lumbalen BSV ist 6-mal häufiger als im zervika- len Bereich. Ein thorakaler BSV zählt zu den seltenen Indikationen. $\mathrm{Zu}$ den degenerativen Wirbelsäulenerkrankungen gehören jedoch auch die osteochondrotischen Veränderungen, die spinalen Stenosen, Gefügestörungen und auch die statisch bedingten Facettarthropathien, bei denen nach ausgeschöpfter konservativer Therapie eine operative Lösung gesucht wird.

In Anbetracht des Kostendrucks im Gesundheitswesen besteht die Notwendigkeit, möglichst viel Abklärung in den vorbereitenden ambulanten und vorstationären Zeitraum zu verlagern, sofern nicht eine akute Schmerzexazerbation eine stationäre Aufnahme zur Schmerztherapie erforderlich macht. Bei einem Patientengut, das auch betagte Patienten mit Komorbiditäten aufweist, wird eine strukturierte Vorbereitung und Planung nicht nur die Zufriedenheit der Patien- 
ten, sondern auch die Dauer der stationären Behandlung verbessern.

Unter dem Grundsatz „so wenig wie möglich - so viel wie nötig“" wird der Umfang des zu planenden Eingriffs, abgesehen von der allgemeinmedizinischen Kondition des Patienten, auch daran zu messen sein, in welchem Umfang eine Beschwerdebesserung mit extensiveren, somit auch riskanteren Verfahren erkauft werden darf. Mitunter wünschen die Patienten bereits selbst keine „Restitutio ad integrum“, sondern sind mit einer Verbesserung der Schmerzsymptomatik und einer Erweiterung des Aktionsradius als spürbare Verbesserung der angestrebten Lebensqualität zufrieden. Aus psychologischer Sicht ist der Spagat zwischen einer optimistisch begleitenden Beratung und einer objektiven Einschätzung des als möglich zu Erreichenden nicht immer einfach. Es muss verhindert werden, dass der Patient das Vorurteil internalisiert, nach einem Eingriff weiterhin schmerzhaft „krank“ zu sein. Auf der anderen Seite wird die Akzeptanz des Erreichten bei objektiver Einschätzung des Möglichen besser sein.

Nur etwa $15 \%$ aller Rückenschmerzen haben spezifische Ursachen, deren Korrelat speziell behandelt werden muss. Trotzdem wurde die DRG 5-831 (Exzision von erkranktem Bandscheibengewebe) 2009 über 161000-mal verschlüsselt und nimmt somit zahlenmäßig den Platz 15 bei den vollstationären Operationen ein.

\section{Begleitmedikation und Begleiterkrankungen}

Ein Abschätzen des Risikopotenzials ist ein Bestandteil der Aufklärung. Neben der Notwendigkeit, die häufig diffusen Beschwerden einzelnen Veränderungen zuzuordnen, ergeben sich insbesondere Probleme eines vorwiegend älteren $\mathrm{Pa}$ tientenklientels. Die allgemeine OP-Fähigkeit muss gegeben sein und wird notfalls durch eine spezielle internistische bzw. auch anästhesiologische konsiliarische Abklärung erhärtet. Bei höheren, z.B. kardiologischen, Risiken ist der zu erwartende Nutzen gegen das Behandlungsrisiko abzuwägen. Eine zunehmend große Gruppe der Patienten mit degenerativen WS-Erkrankungen wird antikoaguliert, wobei die unterschiedlichen Indikationen hierzu zu einer Medikation von Marcumar oder Plättchenaggregationshemmern führen. Während eine solche Behandlung noch bis vor einigen Jahren als generelle Kontraindikation ge- gen diverse operative Vorhaben galt, so mehren sich zuletzt die Meinungen, dass viele Eingriffe auch unter einer modulierten Gerinnung durch Plättchenaggregationshemmer durchgeführt werden können mit der Ausnahme von Operationen im Glaskörper, dem zentralen Nervensystem und bei Eröffnung des Spinalkanals [1]. Hier ist eine Normalisierung vor Operationsbeginn anzustreben. Außer in Notfallsituationen, bei denen einer ASS-Medikation durch Minirin gegengesteuert werden kann, ist hier ein Absetzen der ASS- oder Clopidogrel-Medikation vorzunehmen, ggf. mit Überbrückung durch eine subkutane Heparinisierung. In einer Umfrage unter neurochirurgischen Kliniken wird vor spinalen Eingriffen eine ASS- bzw. Clopidogrelpause von im Mittel 7 Tagen eingehalten [5]. Beide Präparate wirken nicht kompetitiv und die Wirkung schwächt sich infolge der normalen Thrombozytenneubildung ab. Die auch unter Vitamin-K-Gabe undulierenden INR-Werte in der Ausgleichsphase nach Marcumartherapie sind bekannt. Eine Sonderstellung bilden sicherlich die Patienten, die kurz zuvor einen Stent bekommen haben. Das Risiko eines Gefäßverschlusses nach Absetzen von PlAggHemmern wird in der frühen Phase als vermehrt angesehen und muss mit berücksichtigt werden [1].

Für die präoperative Routine bei Patienten, die aufgrund eines Diabetes mellitus Metformin einnehmen, sonografische Untersuchungen bei einer absoluten Arhythmie zum Ausschluss von Vorhofthromben, Feststellung einer AVK der Beine bei ggf. erforderlicher Versorgung mit Antithrombosestrümpfen oder ein Hepatitis/HIV-Screening hat jedes Haus eigene Standards.

In Notfallsituationen kann einer ASS-Medikation durch Minirin gegengesteuert werden. Bei elektiven Eingriffen empfiehlt sich ein Absetzen einer ASS- oder Clopidogrel-Medikation, ggf. mit Überbrückung durch eine subkutane Heparinisierung. In einer Umfrage unter neurochirurgischen Kliniken wird vor spinalen Eingriffen eine ASS-bzw. Clopidogrelpause von im Mittel 7 Tagen eingehalten.

\section{Ätiologie}

Normalerweise beginnen ab dem 35.40. Lebensjahr die degenerativen Prozesse der Wirbelsäule [3]. Vor allem auch eine genetische Disposition, lang anhaltende Haltungsschäden, eine prädisponierende beruflich bedingte Mehrbelas- tung führen zu Verschleißerscheinungen mit Verschiebung des Verhältnisses von Kollagen 1 in Kollagen 2 in den Bandscheiben, zunehmender Fibrosierung, vermindertem Wassergehalt, zunehmendem Stabilitätsverlust und letztendlich pseudoreparativen Vorgängen wie ligamentären Hypertrophien, insbesondere der Ligg. flava und spondylotischen Kantenausziehungen. Je nach Lage finden sich knöcherne Anbaureaktionen im Facettgelenksbereich als auch osteochondrotische Kantenausziehungen. Bildgebende Korrelate sind in der MRT eine Verminderung des Wassergehalts der Bandscheiben in den T2-Sequenzen, Anuluseinrisse, ein Vorwölben der Bandscheiben, insbesondere von Anteilen des Anulus fibrosus als auch gedeckte, perforierende oder sequestrierende Bandscheibenvorfälle mit Nucleus-pulposusAnteilen, Verdickungen von ligamentären Strukturen, Strukturlockerungen mit Wirbelgleiten, Defekte und Sklerosierungen der Endplatte, radiologisch durch die Veränderungen nach Modic klassifiziert $[3,7]$.

\section{Differenzialdiagnostische Erwägungen}

Traumata, entzündliche Spondylolitiden und Spondylodiszitiden wie auch Frakturen mit oder ohne osteoporotische Kalksalzminderung gehören zu den gängigen, nicht degenerativen Differenzialdiagnosen.

Die differenzialdiagnostische Zuordnung der für den Patienten im Vordergrund stehenden Beschwerden zu den häufig komplexen und auch mehrsegmentalen Veränderungen beeinflusst selbstverständlich die Indikationsstellung zu den unterschiedlichen therapeutischen Manövern. Insbesondere im Bereich der LWS sind pseudoradikuläre Schmerzsyndrome aus Facettgelenken oder ISG-Blockaden nicht immer zwanglos von den Symptomen der Nervenwurzelkompressionssyndrome zu differenzieren. Insbesondere die pseudoradikuläre Schmerzausbreitung aus Facettgelenken oder den ISG kann einen radikulären Schmerz imitieren. Im Zweifelsfall kann eine Testanästhesie der Facettgelenke zur Ursache weiteren Aufschluss geben. Möglich sind hier eine Instillation von Lokalanästhetika wie z.B. Ropivacain 7,5\% oder eine Blockade des R. dorsalis des Spinalnervs. Aufgrund der biradikulären Versorgung der Facettgelenke muss hier jeweils in beiden angrenzenden Höhen injiziert werden. Um eine Zuordnung von Schmerzen zu einer speziellen Wur- 
zel zu erreichen, eignet sich die periradikuläre Injektion, z.B. CT-gesteuert, bei der das applizierte Lokalanästhetikum dann bei idealer Platzierung auch das hintere Längsband und die muskulären Äste zur parallel hartgespannten Muskulatur erreicht und ein Circulus vitiosus aus Hartspann und der dadurch bedingten segmentalen Mehrbelastung unterbrochen werden kann. Ggf. kann bei den Punktionen zugleich ein kristallines Cortisondepot mit verabreicht werden. Der Aussagewert der Diskografie mit Erzeugen des „Memory-Pain“, eines diskogenen Schmerzes, ist auch wegen des bestehenden Risikos der Diszitis nicht unumstritten.

\section{Operationsindikation}

Abgesehen von echten Notfällen wie einer Kauda-Symptomatik beim lumbalen Bandscheibenvorfall oder einer hochgradigen Parese stellt sich diese Empfehlung bei den Patienten in der Regel, wenn eine suffiziente konservative Therapie keine zufriedenstellende Besserung erreicht hat und der Leidensdruck des Patienten durch Schmerzen und die Einschränkung der Lebensqualität in einer sinnvollen Relation mit dem zu erwartenden operativen Erfolg gesehen werden kann. Dazu gehört im Optimalfall die positive Korrelation der Beschwerden und der klinischen Untersuchungsbefunde mit der Bildgebung [2]. Im Einzelnen ist zu entscheiden, in welcher Weise schmerzauslösende statische Veränderungen oder direkte Nervenwurzelkompressionssyndrome vorliegen. Sonderfälle sind die zervikale Myelopathie oder die Spinalkanalstenose [6]. Der Schmerz und der dadurch hervorgerufene Leidensdruck ist eine emotional gefärbte Komponente, sodass der Untersucher die Beteiligung von heftigem Schmerzerleben bis hin zu somatoformen Schmerzstörungen auf der einen Seite und Dissimulationstendenzen auf der anderen mit respektieren muss.

Außer bei Notfällen wie einer KaudaSymptomatik besteht eine OP-Indikation, wenn die erreichbare Verbesserung der Lebensqualität in einer sinnvollen Relation zu dem zu erwartenden operativen Erfolg gesehen werden kann. Dazu gehören die positive Korrelation der Beschwerden mit der Bildgebung, die Beurteilung des emotionalen Schmerzerlebens und die Abschätzung des allgemeinen perioperativen Risikos.

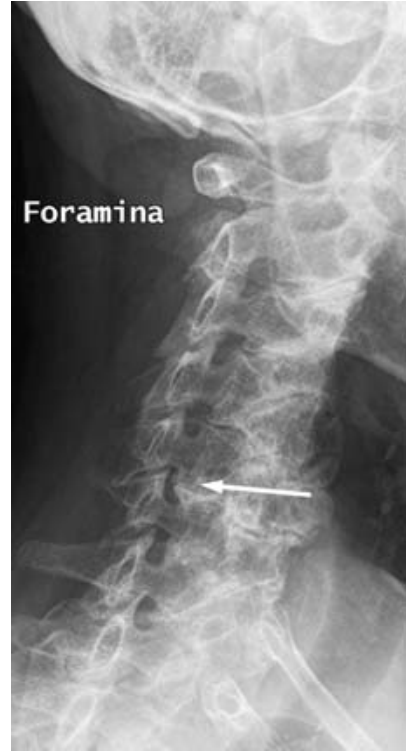

Abb. 1 Die angulierte konventionelle Röntgendarstellung der HWS dokumentiert die ausgeprägte knöcherne Foramenstenose HWK 5/6 mit fakultativer Nervenwurzelkompression C6.

\section{Radiologische Diagnostik}

Konventionelle Röntgenbilder dienen im Wesentlichen der Darstellung von Haltungsabnormalitäten und spondylotischen Kantenausziehungen. Insbesondere im zervikalen Bereich haben sie noch ihre Berechtigung, nachdem die MRT aufgrund höherer Detailgenauigkeit und Darstellbarkeit der neuralen Strukturen die Durchführung einer Computertomografie weitgehend ersetzt hat. Foraminal einengende Kantenausziehungen mit fakultativer Nervenwurzelkompression werden durch angulierte Foramenaufnahmen aufgedeckt (Abb. 1). In gleicher Weise werden segmentale Instabilitäten durch Funktionsaufnahmen sichtbar und geben so im Vorfeld Hinweise auf eine ggf. zu planende instrumentierte Stabilisierung.

Den Standard bei der Diagnostik bildet derzeit die Kernspintomografie. Eine CT ist ggf. zur Beurteilung der knöchernen Strukturen und als sinnvolle Ergänzung nach Durchführung einer Myelografie zu erwägen. Die Myelografie hat ihre Berechtigung immer noch bei Veränderungen, die in der MRT nicht zugeordnet werden können. Aufnahmen in Funktionsstellungen und insbesondere bei Rezidivproblematiken bilden die häufigen Indikationsstellungen (Abb. 2). Es bleibt abzuwarten, inwieweit die offene MRT hier weiterhelfen kann, die jedoch

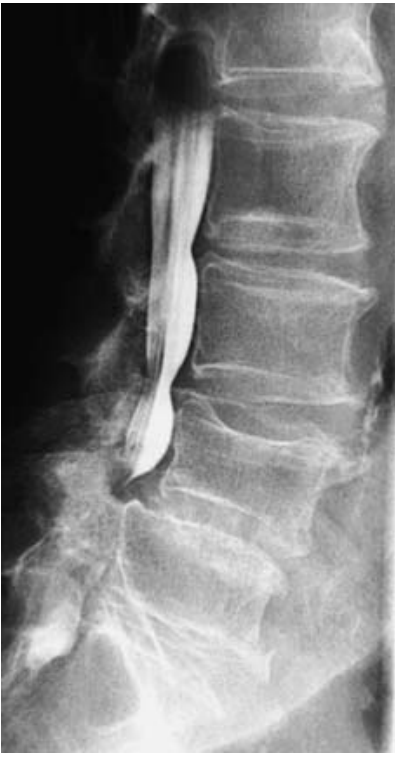

Abb. 2 Konventionelle Myelografie: Inkompletter Stopp des Kontrastmittelbands bei L4/5 bei ansonsten noch ausreichender Weite des Spinalkanals als Ursache einer belastungsabhängigen Claudicatio spinalis bei Spinalkanalstenose und segmentaler Gefügelockerung $L 4 / 5$.

noch nicht flächendeckend verfügbar ist. $\mathrm{Zu}$ beachten ist ebenfalls eine Schrittmacherimplantation als Kontraindikation gegen eine MRT.

\section{Degenerative Veränderungen der HWS}

Letztendlich stehen Nervenwurzelkompressionssyndrome zahlenmäßig im Vordergrund der operativen Diagnosen. Hierbei sind ätiologisch sog. „weiche“ Bandscheibenvorfälle mit fast ausschließlicher Extrusion oder Sequestrierung von Bandscheibenmaterial, die häufiger bei jüngeren Patienten auftreten, gegenüber den „harten“ Vorfällen in der Auswirkung gleichwertig (Abb.3). Sog. „harte“ Bandscheibenvorfälle weisen regelmäßig auch eine Bandscheibenvorwölbung auf, das eigentliche Substrat der Wurzelkompression generiert sich aus insbesondere foraminalen Stenosen durch osteochondrotische Kantenausziehungen [9]. Dadurch ist die Anamnese der Schmerzhaftigkeit häufig auch länger als beim reinen Bandscheibensequester, der einen akuten Schmerzbeginn verursacht.

Das operative Ziel ist in beiden Fällen die Dekompression der austretenden Nervenwurzel. Nicht selten sind in der Bildgebung mehrere Segmente morphologisch alteriert, sodass nach der Klinik entschieden werden muss, welches Segment für die beim Patienten im Vorder- 

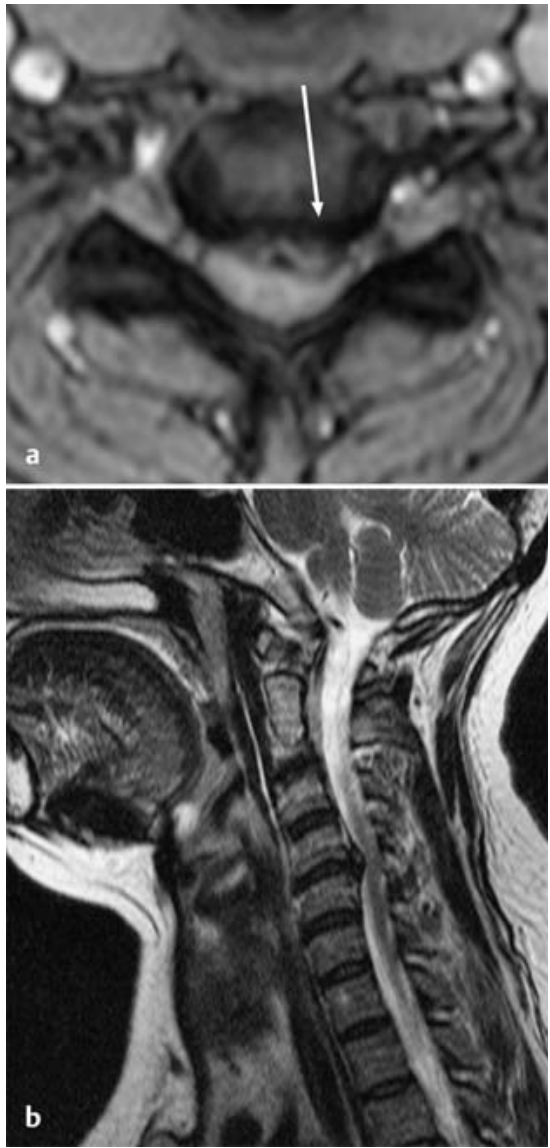

Abb. $\mathbf{3} \mathbf{a}$ und $\mathbf{b}$ Darstellung eines zervikalen BSV HWK 4/5 in der axialen (a) und sagittalen (b) MRT-Schichtung. Die klinische Symptomatik entsprach eher einem C7- und C8-Syndrom mit leichten Paresen, die sich nach operativer Dekompression besserten. Der Patient wurde schmerzfrei entlassen.

grund stehenden Beschwerden angeschuldigt werden muss. Häufig ist die die Lebensqualität einschränkende Symptomatik behebbar, wenn nur dieses Segment operiert wird. $\mathrm{Zu}$ beachten sind jedoch auch die Nachbarsegmente. Bei schon weit fortgeschrittenem Verschleiß ist zu diskutieren, ob angesichts der zu erwartenden Beschwerden aus dem Nachbarsegment dieses nicht mit in die Operationsplanung einbezogen werden sollte. Die radikuläre Zuordnung folgt den bekannten Schmerzausbreitungen in die Dermatome ggf. mit Sensibilitätsstörungen und Reflexdifferenzen und in schwereren Fällen durch Präsentation von Paresen der Kennmuskulatur. Einfach zu erkennen ist die Bizepsparese bei einer C6-Läsion, die Trizepsparese der C7-Alteration und die Schwäche der kleinen Handmuskulatur bei C8-Syndrom. Hier ist die wichtigste Differenzialdiagnose das Sulcus-ulnaris-Syndrom, bei dem Sensibilitätsdifferenzen

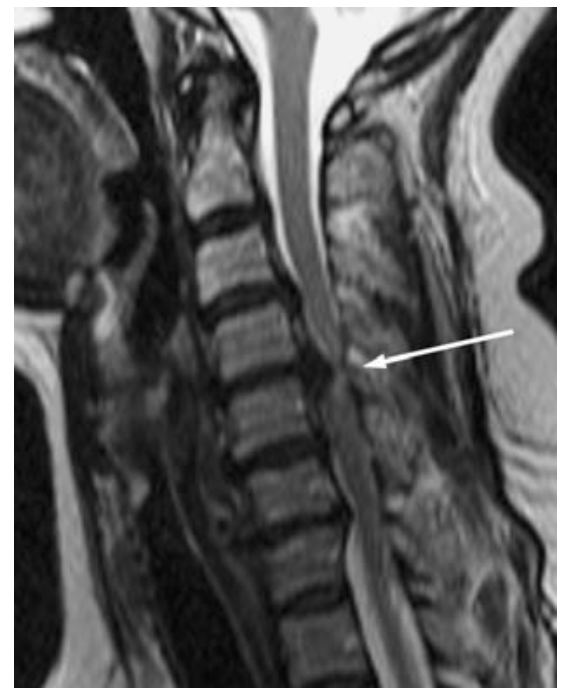

Abb. 4 Die aus Osteochondrosen und einem BSV HWK 5/6 gebildete, diskogene Raumforderung im Spinalkanal hat zu einer zervikalen Myelopathie geführt. Klinisch bestehen keine wegweisenden radikulären Beschwerden, sondern Zeichen der langen Bahnen mit einem spastisch-ataktischen Gangbild. Eine leichte Hyperintensität im Myelon muss als Korrelat der Rückenmarksaffektion gesehen werden. Es besteht die absolute OP-Indikation, um ein Fortschreiten der myelären Symptomatik zu verhindern.

zwischen der ulnaren und radialen Seite des Ringfingers gefunden werden können, die bei einem C8-Syndrom in der Regel fehlen. Bei nicht eindeutigen Befunden kann durch eine Bestimmung der Nervenleitgeschwindigkeit über dem Sulcus ulnaris eine Klärung herbeigeführt werden. Die Elektromyografie kann in den Kennmuskeln einen Hinweis auf neurogene Umbauveränderungen geben, wobei eingeschränkt beachtet werden muss, dass auch metamere Abweichungen vorkommen können und eine neurale Schädigung erst nach Ablauf von etwa 3-4 Wochen im EMG verwertbare Befunde ermöglicht.

Bei medianen, v.a. osteochondrotischen Ausziehungen mit Myelonkontakt kann sich das Bild der sog. zervikalen Myelopathie [9] entwickeln (Abb. 4). Hier stehen die radikulären Beschwerden im Hintergrund, sie können mitunter auch ganz fehlen. Das Krankheitsbild wird geprägt von Störungen der langen Bahnen, wobei sowohl eine sich langsam entwickelnde spinale Spastik als auch ataktische Störungen das Gangbild und v.a. die Gangsicherheit des Patienten verschlechtern. Es kommt häufiger zu Sturzereignissen, in deren Rahmen der osteochondrotische Knochensporn weitere, mitunter akute Verschlechterungen der Neurologie hervorrufen kann bis hin zum Querschnittssyndrom. Aus diesem Grunde wird man den Patienten bez. einer OP-Indikation aggressiver beraten und bei ausgeprägten Veränderungen in der bildgebenden Diagnostik zur Operation raten, bevor sich gravierende neurologische Störungen abzeichnen, die nur eine begrenzte Rückbildungstendenz aufweisen.

Je nach gewähltem operativen Verfahren sollte bereits vor der Operation eine zumeist weiche Zervikalstütze angepasst werden. Da der Patient diese für einige Wochen tragen wird, trägt die gewählte Passform, Höhe und Weite der Stütze zur Compliance des Patienten bei.

Im Zweifelsfalle kann die Neurophysiologie bei der Beurteilung radikulärer Schäden weiterhelfen. Das EMG ist in der Regel bereits nach 3-4 Wochen verändert.

\section{Lendenwirbelsäule}

Die in der täglichen Praxis häufigste Form zur Operation anstehender degenerativer Erkrankungen ist der Bandscheibenvorfall bzw. die dadurch hervorgerufene radikuläre Symptomatik. Er ist Ausdruck der Veränderungen der Bandscheibenmatrix selbst. In fortgeschrittenem Wirbelsäulenalter kommen weitere einengende Komponenten hinzu, die als knöcherne Kantenausziehungen der Grund- oder Deckplatte insbesondere foraminal den Wurzelverlauf einengen können [8]. Andere Prädilektionsstellen sind die Facettgelenke, bei deren Hypertrophie der Recessus lateralis verengt wird, in dem die Wurzel vor dem foraminalen Verlauf gelegen ist. Verdickungen des Lig. flavum bilden häufig in Kombination mit hypertrophierten Facettgelenken das Korrelat der Claudicatio spinalis bei engem Spinalkanal [6]. Je nach lokalem Überwiegen der Einengung wird zwischen einer zentralen und einer lateralen Spinalkanalstenose differenziert (Abb. 5). Die genannten Hypertrophien sind Ausdruck der beginnenden Segmentinstabilität, die auch weit fortgeschritten sein kann, bis hin zu radiografisch nachweisbaren Pseudospondylolisthesen.

Bei dem einfachen Bandscheibenvorfall reicht in der Regel die MRT zur Zuordnung der Beschwerden. Insbesondere in den T2-gewichteten Sequenzen lassen sich die bandscheibenbedingten Wurzelkompressionen gut nachvollziehen und differenzialdiagnostisch von medial pro- 
labierten Synovialzysten der Facettgelenke unterscheiden (Abb.6). Diese können eine beachtliche Größe entwickeln und selbst eine schmerzhafte Wurzelkompression hervorrufen. Sinnvoll ist in jedem Falle eine Darstellung der Wirbelsäule auch in der sagittalen Schnittführung bis extraforaminal, um Vorfälle aufzudecken, die hier in etwa 7\% der Fälle lokalisiert sind (Abb. 7). Der operative Zugang wäre hier nicht interlaminär über eine Flavektomie, sondern über einen extraforaminalen Zugang unter Schonung der Facettgelenkskette zu planen. Intraforaminale Vorwölbungen der Bandscheiben sind relativ frequent, haben jedoch nur teilweise entscheidenden Krankheitswert. Die Kompression der abgehenden Wurzel lässt sich v.a. in den sagittalen Schichten gut beurteilen oder unwahrscheinlich machen. Mitunter sind anlagebedingte Störungen des lumbosakralen Übergangs mit z.B. Lumbalisierung des SWK1 Ursache einer vermeintlichen Differenz zwischen Schmerzausbreitungsgebiet und segmentaler Zuordnung im MRT. Differenzialdiagnostische Probleme können auch aufgrund einer metameren Variabilität entstehen.

Pseudoradikuläre Beschwerden können in ihrer Ausprägung von der Schmerzausbreitung ein radikuläres Syndrom imitieren. Ein pseudoradikuläres Schmerzsyndrom aus einer ISG-Blockierung kann wie ein S1-Syndrom imponieren mit einer Ausbreitung über das Gesäß in den dorsalen Ober- und teilweise Unterschenkel. Die pseudoradikulären Beschwerden aus Blockierungen der Facettgelenke können ebenfalls in die dorsalen Anteile ausstrahlen, häufig ist aber auch eine Ausbreitung in die Leistenregion und von hier aus dann dem ventralen Oberschenkel in den ventralen Unterschenkel folgend mit Kreuzen mehrerer Dermatomareale. Die Ausbreitungslokalisationen sind intraindividuell gut und ortsgebunden reproduzierbar, weisen jedoch eine hohe interindividuelle Variabilität auf, die einen verlässlichen Rückschluss auf die Läsionsursache behindert. Bei pseudoradikulären Beschwerden fehlen regelhaft Paresen. Sensibilitätsstörungen bis zu Parästhesien im Schmerzareal sind ebenfalls selten, aber nicht ausschließbar.

Radikuläre Beschwerden beinhalten typischerweise die Trias aus dermatomfolgender Schmerzbahn, Hypästhesie und Paresen der Kennmuskeln. Nur in einem kleineren Anteil jedoch finden sich alle

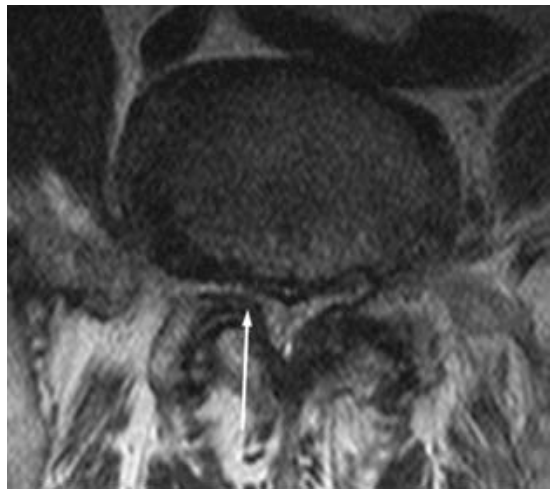

Abb. 5 Komplexe degenerative Veränderung L4/5 mit dem resultierenden Bild der Spinalkanalstenose und Facettarthropathie mit einer knöchernen und ligamentären Einengung des rechten Recessus lateralis (Pfeil) und einer eher zentralen Stenose links. Klinisch findet sich keine typische bds. Claudicatio spinalis, sondern v.a. eine bewegungsabhängige radikuläre Schmerzsymptomatik L5 rechts, die durch die hypertrophierende Einengung erklärt wird. Operativ wurde eine rechtsseitige erweiterte interlaminäre Fensterung mit Dekompression der Rezessusstenose vorgenommen und eine Erweiterung nach links mittellinienübergreifend in Undercutting-Technik. Das postoperative Ergebnis war sehr zufriedenstellend.

diese Symptome. Häufiger stehen Schmerzen und Sensibilitätsstörungen im Vordergrund. Schmerzfreie Paresen bilden die Ausnahme und lassen die Befürchtung eines sog. Wurzeltods zu, insbesondere, wenn die Parese bei nachlassendem Schmerzerleben entstanden ist. Hier ist die Remissionsprognose als we-

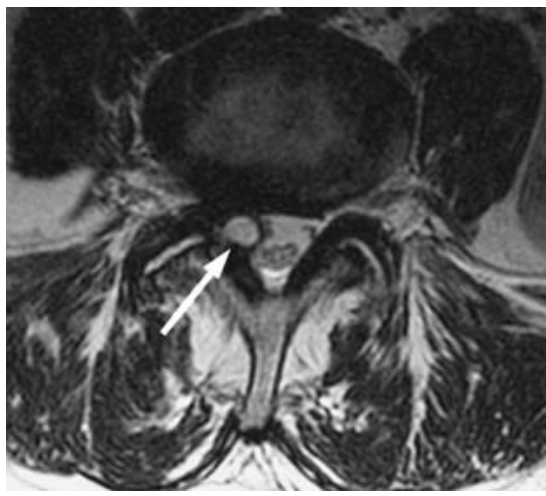

Abb. 6 Synovialzyste des Facettgelenks. In der T2-gewichteten MRT-Sequenz stellt sich die synoviale Aussackung dar, die im Recessus lateralis eine Wurzelkompression verursacht. Die interlaminäre Resektion führte zur kompletten Beschwerdefreiheit.

niger günstig einzustufen. Bei solchen länger bestehenden schmerzfreien und hochgradigen Paresen muss der Patient in der Aufklärung auf die zweifelhafte Remissionschance hingewiesen werden, bevor er sich für die Durchführung eines Eingriffs entscheiden wird.

Radikuläre Bewegungsstörungen sind anhand der Kennmuskulatur ebenfalls gut zuzuordnen. Bei einer Hüftbeugerparese wird man zunächst nach einer L3-Schädigung suchen, bei Quadrizepsparese nach L4- und bei Fußsenkerparese nach einer S1-Kompression. Bei einer Fußheberparese muss differenzialdiagnostisch ein peripherer Schaden des N. peronäus ausgeschlossen werden, der
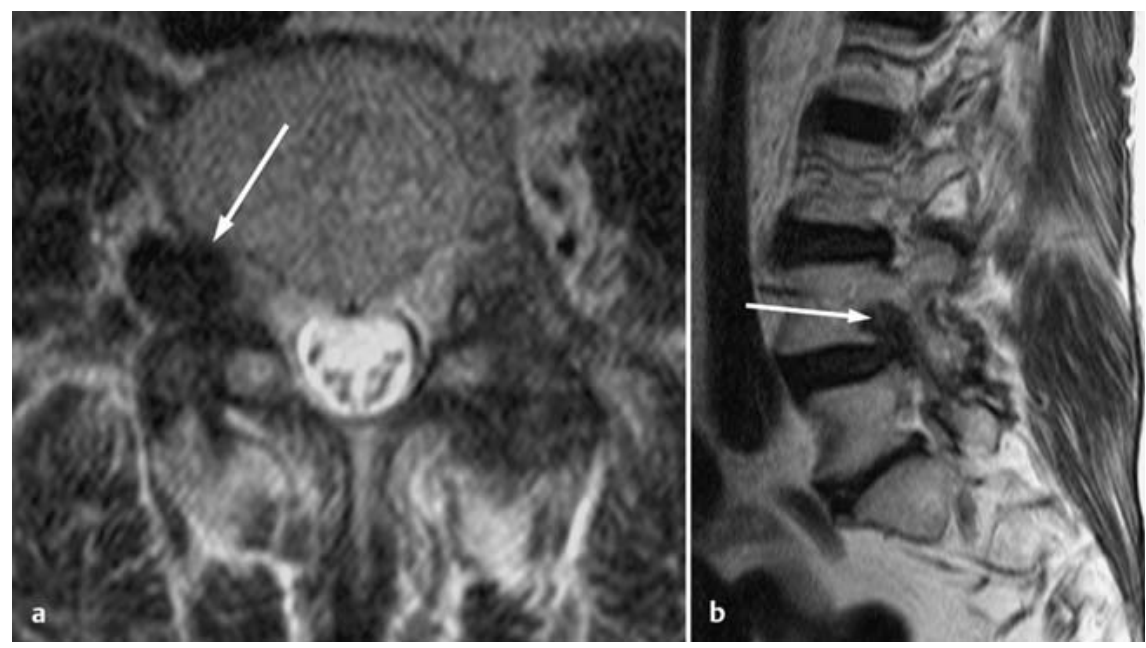

Abb. 7 a und b Abbildung eines extraforaminalen BSV L4/5 (Pfeilmarkierungen) in der axialen (a) und sagittalen (b) T2-gewichtenen MRT-Schnittführung. Klinisch besteht eine akute heftige Symptomatik mit Quadrizepsparese durch Kompression der Nervenwurzel L4 rechts. Insbesondere die Darstellung des sagittalen Bildes belegt die vollständige Verlegung des Foramens. Der Sequester wurde problemlos über einen extraforaminalen Zugang entfernt. Postoperativ sofortige Schmerzfreiheit und rasche Besserung der Parese im Verlauf. 
ebenfalls Schmerzen im Unterschenkel verursachen kann. Bei einer solchen peripheren Parese ist jedoch nie eine Parese des M. glutaeus med. zu sehen, der sich bei der Untersuchung in einem Trendelenburg-Zeichen offenbart. Im Zweifelsfalle hilft auch hier bei länger bestehenden Beschwerden ein EMG des M. glutaeus med. bzw. die Messung der Nervenleitgeschwindigkeit über dem $\mathrm{N}$. peronaeus weiter.

Inwieweit nach ausgedehnteren LWSOperationen mit oder ohne instrumentierte Stabilisierungen ein Mieder verordnet wird, obliegt der jeweiligen Einschätzung des Operateurs. Deutlich im Vordergrund stehende Dorsalgien lenken den Verdacht auf statisch begründete Schmerzursachen, eine schmerzhafte arosive Osteochondrose der Grundund Deckplatte lässt sich entsprechend der Modic-Klassifizierung insbesondere im 1. Stadium durch die MRT aufdecken und ist häufig nur durch eine instrumentierte Stabilisierung erfolgreich zu therapieren.

\section{Nachkontrolle}

Eine Durchführung einer Nachkontrolle nach operativer Behandlung degenerativer Wirbelsäulenveränderungen wird je nach den Möglichkeiten der operativen Einheit und dem Ausmaß des durchgeführten Eingriffs durch die operierende Klinik selbst durchgeführt. Er dient neben der Vertrauensbildung seitens des Patienten selbst auch der eigenen Qualitätskontrolle. Bei weniger eingreifenden Methoden wie einer unkomplizierten Bandscheibenoperation oder einer spinalen Dekompression kann diese ganz unzweifelhaft durch den ambulanten hausärztlichen bzw. fachärztlichen Bereich abgedeckt werden. Bei komplizierteren Eingriffen, insbesondere nach Einbringen von Implantaten ist jedoch eine Nachuntersuchung durch den operativen Bereich sinnvoll, zumindest, um die Einheilung bzw. Durchbauungsvorgänge beurteilen zu können. In Abhängigkeit davon möchte der Patient natürlich informiert werden, wie die Belastungsmöglichkeiten aufgebaut und wann ggf. verordnete Stützmieder oder Zervikalstützen abgelegt werden können.

Insbesondere jedoch kommen auch Patienten zur Nachuntersuchung, bei denen das operative Ergebnis nicht den projizierten Erwartungen entspricht. Hier ist eine Aufklärung über die Mög-
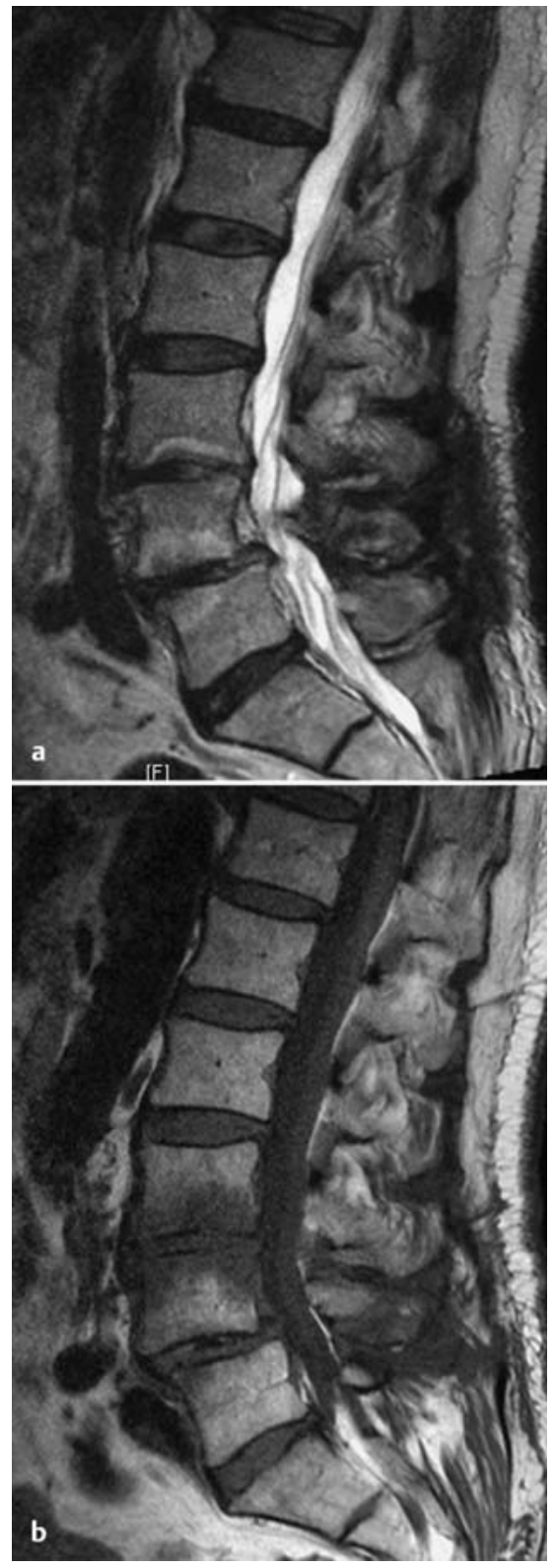

Abb. 8a und b Nach stattgehabter Bandscheibenoperation L4/5 hat sich eine Pseudospondylolisthese ausgebildet. Klinisch finden sich v. a. belastungsabhängige Dorsalgien, gepaart mit einem L4- und einem L5-Syndrom. In der MRT finden sich neben der Gefügelockerung $L 4 / 5$ auch die Zeichen der arosiven Osteochondrose L3/4 und degenerative Veränderungen bei L5/S1. Es wird die Indikation zur instrumentierten Fusion L3 bis S1 gestellt (Implantation eines Fixateur interne plus vordere Abstützung durch Cages).

lichkeiten und Grenzen der operativen Behandlung hilfreich, wenn dies auch nicht immer vom Patienten akzeptiert werden wird. Auch ein Ausloten weitergehender konservativer oder operativer Möglichkeiten kann in diesem Kontext beraten werden. Ein Überdenken der Arbeitsfähigkeit oder die Ergonomisierung des Arbeitsplatzes gehören in diesen Bereich wie auch eine weitere Fortschreibung physikalischer Therapiemaßnahmen, von Krankengymnastik oder Manualtherapie. Bei chronischen Schmerzen ist die Einstellung auf Analgetika bzw. ambulantes Erlernen von Schmerzbewältigungsstrategien denkbar. In diesem Zusammenhang sei auf die verschiedenen Scores hingewiesen, wobei die visuelle analoge Schmerzskala die bekannteste und schnell $\mathrm{zu}$ verwendende ist. Andere Scores wie z.B. der Neck-PainScore oder der Oswestry-Disability-Index sind aufwendiger.

\section{Diskussion}

Wirbelsäulengenerierte Beschwerden und Dorsalgien gehören zahlenmäßig zu den häufigsten Gründen der Konsultierung in der täglichen Praxis. Der Großteil kann durch konservative Maßnahmen erfolgreich behandelt werden [8]. Dabei kommen je nach Ausprägung neben der Beratung und ggf. kurzfristiger Schonung und Wärmeanwendungen auch medikamentöse Strategien, physikalische, aber auch anästhesiologische Maßnahmen in Betracht. Die Frequenz von Infiltrationen bis in den wirbelsäulennahen Bereich hat in den letzten Jahren zugenommen. Bei Nervenwurzelkompressionssyndromen ist der Wert chiropraktischer Manöver nicht ganz unumstritten. Hierunter kann es bei Wurzelkontakt auch zu Exazerbationen kommen.

Außerhalb von reinen, weichen Bandscheibenvorfällen sind die degenerativen Veränderungen ein buntes Bild, das Minderungen der Bandscheibenhöhe, eine abnorme Beweglichkeiten des rigideren Segments, osteochondrotische Kantenausziehungen und arosive Veränderungen von Grund- und Deckplatten, Hypertrophien der Facettgelenke, Verdickung der ligamentären Aufhängung, insbesondere der Ligg. flava, umfasst [3] (Abb. 8). Selten liegen solche Veränderungen isoliert vor, häufiger ist ein Mischbild, wobei zu entscheiden ist, was in erster Linie als Korrelat des Beschwerdekomplexes infrage kommen wird. Neben schmerzhaften statisch generierten Veränderungen sind Kompressionssyndrome zu beachten. Je nach Spezifikum der Veränderungen ist eine größere Zahl von operativen Manövern verfügbar. Neben den reinen Dekompressionen werden auch zunehmend Implantate verwendet, wobei die Diskussion um die Verwendung der funktions- 
erhaltenden Bandscheibenprothese, der dynamischen oder rigiden Stabilisierung, von Zwischenwirbelraumcages aus unterschiedlichen Materialien, der Einbringung von interspinösen Spacern oder einer direkten Verschraubung der Facettgelenke eine lebhafte Basis hat.

Je mehr Segmente auch radiologisch alteriert sind, desto schwieriger wird die Entscheidung, das Ausmaß eines operativen Eingriffs festzulegen. Hier ist der klinische Eindruck über die Belastungsabhängigkeit der Beschwerden, das Überwiegen von Dorsalgien oder Ischialgien oder anamnestische Hinweise zu ggf. bereits erfolgten Testanästhesien oder PRTs häufig hilfreicher als die Bildgebung. Im Zweifelsfalle sind neben den Funktionsaufnahmen zur Aufdeckung von segmentalen Instabilitäten immer noch Funktionsmyelografien wertvoll, die die bewegungsabhängigen Komponenten bei Einengungen des Spinalkanals darstellen. Die angeschlossene Myelo-CT ergänzt die Aussagekraft. Insbesondere an den unteren Extremitäten kommt der Elektrophysiologie ein besonderer Stellenwert zu. Segmentale neurogene Schäden lassen sich ab etwa einem Schädigungszeitraum von 4 Wochen ableiten. Auf der anderen Seite ist eine akute Neurologie bei einem bereits sehr ausgeprägten neurogenen Schaden und Umbau immer Anlass, eine weitere Ursache als Läsionsquelle anzunehmen als die, die den bereits lange vorhandenen Umbau verursacht hat.

Die begleitende Bildgebung und hier insbesondere die Kernspintomografie bilden eine wesentliche Hilfestellung, können jedoch per se nur in seltenen Fällen die Entscheidung zu einem operativen

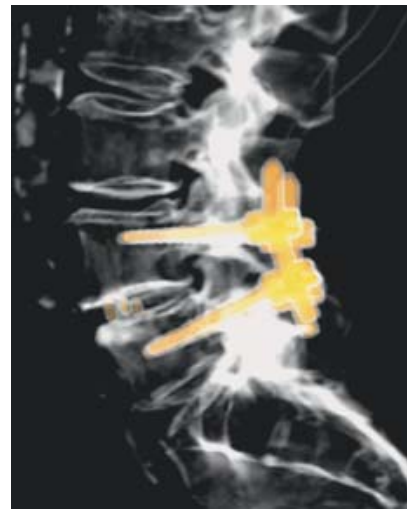

Abb.9 Monosegmentale rigide Stabilisierung mittels transpedikulärem Fixateur interne mit vorderer Abstützung durch einen Cage nach spinaler Dekompression und Reposition einer Pseudospondylolisthese.

Verfahren begründen. Insbesondere bei der Claudicatio spinalis fehlt die reliable Korrelation zwischen klinischem Beschwerdebild und dem Ausmaß der Stenose [4]. In der Regel sollen bildgebende Verfahren nur eine Hilfe bieten, das Bild eines klinischen Syndroms zu erklären und eine Lokalisationsdiagnostik des Schädigungsorts einzugrenzen. Die klinische Relevanz einzelner MRT-Befunde wie die der aktivierten Osteochondrose nach den Einteilungen von Modic [7], kernspintomografisch dokumentierbare Anuluseinrisse oder die „black disc“ sind lediglich radiologische Beschreibungen, die mit klinischer Relevanz korreliert werden müssen. Bei einem Überwiegen von Dorsalgien und dem Hinweis auf degenerative Instabilitäten bzw. ausgeprägte aktivierte Osteochondrosen ist neben der Dekompression die Fusionierung zu erwägen (Abb. 9).

\section{Literatur}

1 Chassot PG, Marcucci C, Delabays A et al. Perioperative antiplatelet therapy. Am Fam Physician 2010; 82: 1484-1489

2 Diemer W, Burchert H. Chronische Schmerzen - Kopf- und Rückenschmerzen, Tumorschmerzen. Gesundheitsberichterstattung des Bundes. Themenhefte 2002; 7

${ }^{3}$ Fardon DF, Milette PC. Nomenclature and classification of lumbar disc pathology. Spine 2001; 26: E93-E113

${ }^{4}$ Feldmann PH. Diagnostik und Therapie der lumbalen Spinalkanalstenose. Orthopädie Mitteilungen 2003; 2: 136-140

${ }^{5}$ Korinth MC, Gilsbach JM, Weinzirl MR. Lowdose aspirin before spinal surgery: results of a survey among neurosurgeons in Germany. Eur Spine J 2007; 16: 365-372

${ }^{6}$ Lorenz M, König K Rickels $E$. Klinik und Therapie der Claudicatio spinalis bei Spinalkanalstenosen. OP-Journal 2009; 25: 124-128

7 Modic MT, Ross JS. Lumbar degenerative disk disease. Radiology 2007; 245: 43-61

8 Pingel A, Scholz M, Kandziora F. Operative Behandlung des lumbalen Bandscheibenvorfalls. OP-Journal 2009; 25: 112-117

${ }^{9}$ Rickels E, König K, Lorenz $M$ et al. Klinik und operative Therapie des zervikalen Syndroms. OP-Journal 2009; 25: 82-86

10 Statistisches Bundesamt. Diagnosedaten der Patienten und Patientinnen in Krankenhäusern (einschl. Sterbe- und Stundenfälle). Gesundheit, Fachserie 12; Statistisches Bundesamt, Wiesbaden 2009, http://www.gbebund.de: Diagnosedaten der Krankenhäuser ab 2000

\section{Prof. Dr. med. Martin Lorenz}

Oberarzt

Prof. Dr. med. Eckhard Rickels

Leitender Arzt

Dr. med. Kathrin König

Oberärztin

Bereich Neurochirurgie

Klinik für Unfallchirurgie, Orthopädie und Neurotraumatologie des AKH Celle Siemensplatz 4

29223 Celle

martin.lorenz@akh-celle.de 